\title{
Estudio de la distribución de poros activos y totales en membranas cerámicas planas de microfiltración
}

\author{
L. PALACIO, P. PRÁDANOS, J. I. CALVO, A. HERNÁNDEZ, C. ALMANDOZ ${ }^{1}$, M. AMARAL ${ }^{1}$ Y J. MARCHESE ${ }^{1}$ \\ Dpto. Termodinámica y Física Aplicada, Facultad de Ciencias, Universidad de Valladolid, 47071 Valladolid, España \\ ${ }^{1}$ Centro Regional de Estudios Avanzados Científicos y Tecnológicos (CREACYT) Laboratorio de Ciencia de Superficies y Medios Porosos (UNSL), \\ c.c. 256, 5700 San Luis, Argentina
}

\begin{abstract}
En este trabajo se ha estudiado la influencia del tamaño de partículas y de la temperatura de sinterizado en la preparación de membranas planas de microfiltración. Estas membranas se prepararon a partir de materias primas cerámicas controlando, entre otras variables: el tamaño de partículas, la formulación química de la pasta y su tratamiento térmico. La morfología estructural de las membranas en función del tamaño de partícula y la temperatura de sinterizado ha sido estudiada mediante técnicas de porosimetría de mercurio y porometría de aire-líquido. Las dos técnicas de análisis han sido utilizadas por su complementariedad, puesto que la porosimetría de mercurio informa sobre los poros totales, mientras que la porometría de aire-líquido lo hace sobre los poros activos. Los resultados muestran que tanto el tamaño de los poros activos como de los totales, se incrementa con el tamaño de partícula de la pasta empleada. De forma similar, el incremento de la temperatura de sinterizado (dentro del rango de trabajo) produce un aumento del tamaño medio de poro y un ensanchamiento de la distribución.
\end{abstract}

Palabras clave: Materiales porosos, Membranas cerámicas, Síntesis de membranas planas, Microfiltración, Temperatura de sinterizado.

\section{Study of the pore size distribution of active and total pores in microfiltration ceramic flat membranes}

In this work, it has been studied the influence of the particles size and sinterization temperature in the preparation of flat microfiltration ceramic membranes. These membranes were made from ceramic materials by controlling, among other factors, the particle size, the chemical formulation of the paste and its thermal treatment. The structural morphology of the membranes has been studied as a function of particle size and sinterization temperature, by using mercury porosimetry and air-liquid porometry. Both techniques have been used due to their complementarity, as far as mercury porosimetry gives information on total pores, while air-liquid porometry does it on active pores. Results showed that both active and total pores size, are incremented with the size of the used particles. Similarly, the increase of the sinterization temperature (within the range of work) results in an increase of the mean pore size and a broadening of the distribution.

Keywords: Porous materials, Ceramic membranes, Flat membranes synthesis, Microfiltration, Sinterization temperature

\section{INTRODUCCIÓN}

La viabilidad económica de los procesos de separación por membranas depende del grado de separación conseguido en el proceso, de la permeabilidad y del tiempo de vida de la membrana utilizada. Para la mayoría de dichos procesos, sobre todo aquellos en que la fuerza impulsora es un gradiente de presión, existen gran número de membranas comerciales, poliméricas o cerámicas, que permiten conseguir factores de recuperación similares o superiores a los convencionales (1). Por otra parte, los procesos de separación con membranas tienen la ventaja de su bajo consumo energético y la posibilidad de trabajar a bajas temperaturas, frente a procesos convencionales como la evaporación. No obstante, en muchos casos, el tiempo de vida de estas membranas puede hacer que estos procesos no sean viables desde el punto de vista económico. Las membranas cerámicas han contribuido en gran medida a solventar este problema. Sobre todo debido a una serie de factores como son, la mayor permeabilidad que se traduce en que el área de filtración requerida sea significativamente menor; y la alta resistencia térmica, química y mecánica que permiten una recuperación casi total de este tipo de filtros (2). Sin embargo, el costo inicial de las membranas cerámicas hace que en muchos procesos sean desestimadas.

Los altos costes de las membranas cerámicas se deben sobre todo a que, para obtenerlas con las mismas características selectivas que las membranas poliméricas de ultrafiltración o nanofiltración, es necesario recurrir a partículas con una distribución de tamaños estrecha y la utilización de procesos de sol gel. No obstante, las membranas cerámicas de microfiltración de alta calidad pueden ser obtenidas con los métodos tradicionales de fabricación de cerámicas, pudiendo competir en coste y selectividad con las poliméricas.

Independientemente del tipo de módulo (configuración externa de la membrana) con el que se esté trabajando, la configuración interna de las membranas cerámicas es similar 
a las poliméricas; es decir, están formadas por dos o más capas. Así consisten en una o varias capas de soporte poroso, que suelen estar fabricadas de materiales baratos y con procesos convencionales y una capa selectiva mucho más elaborada. Dado que la estructura de la membrana va ha determinar las características de operatividad de ésta, el proceso de fabricación y los materiales empleados en su fabricación son de gran importancia $(3,4)$.

En este trabajo se han fabricado membranas planas de microfiltración utilizando el método de colada, con pastas que normalmente son usadas para la fabricación de la capa activa de membranas de microfiltración. El tamaño de poro de la membrana viene determinado principalmente por el tamaño de las partículas de la pasta; no obstante, la temperatura de sinterizado puede variar significativamente tanto el valor medio de la distribución como la anchura de ésta.

Este estudio ha intentado analizar como influyen estos dos factores, (distribución de tamaños de las partículas empleadas y temperatura de sinterizado), en la distribución de poros activos y totales de la membrana, y por tanto en sus características de retención.

\section{MATERIALES Y MÉTODOS EXPERIMENTALES}

\section{Síntesis de las membranas}

\subsubsection{MATERIAS PRIMAS}

Las materias primas utilizadas en la preparación de las pastas cerámicas del presente trabajo fueron arcilla, cuarzo y alúmina. Entre las propiedades físicas de la arcilla son de destacar su módulo de rotura en verde entre $30-40 \mathrm{Kg} / \mathrm{cm}^{2}$ determinado por un sistema de pruebas de Tensión/Compresión de acuerdo a normas ASTM; superficie específica de 17 - $22 \mathrm{~m}^{2} / \mathrm{g}$ determinada por adsorción de Nitrógeno (método BET); capacidad de intercambio catiónico entre 6 - 8 meq A.M./ 100 gramos; contracción en verde entre el 6 - 8\%. La distribución de tamaño de partículas estuvo comprendida entre 0.2-200 $\mu \mathrm{m}$, con un tamaño medio representativo de $2.7 \mu \mathrm{m}$.

La alúmina $\left(\mathrm{Al}_{2} \mathrm{O}_{3}\right)$ empleada en la elaboración de las pastas es alúmina calcinada obtenida a partir de bauxita mediante el proceso complejo Bayer, con un contenido químico típico mayor al $98 \%$ en $\mathrm{Al}_{2} \mathrm{O}_{3}$, determinado por ICP, $0,5-1 \%$ de $\mathrm{Na}_{2} \mathrm{O}$ y $0,07-0,05 \%$ la suma de $\mathrm{SiO}_{2}$ con $\mathrm{Fe}_{2} \mathrm{O}_{3}$. Las características físicas típicas de esta alúmina corresponden a una densidad aparente de $1,0 \mathrm{~g} / \mathrm{cm}^{3}$ y densidad de empaquetamiento de $1,2 \mathrm{~g} / \mathrm{cm}^{3}$, determinado por ASTM -B-527-81, área superficial por BET de $60 \mathrm{~m}^{2} / \mathrm{g}$ y un análisis de tamizado con tamaños de mallas estándar Tyler de un $5-15 \%$ de partículas retenidas en malla 100, un $65-75 \%$ retenidas en malla 200 y un 90 - $97 \%$ retenidas en malla 325 .

El cuarzo contiene, principalmente $\mathrm{SiO}_{2}$ en el rango del 95 al $98 \%$ en peso, cantidades menores de $\mathrm{Al}_{2} \mathrm{O}_{3}$, entre $0,5-1 \%$, y bajo contenido en álcalis $(0,1-0,3 \%)$. La sílice presente en el cuarzo en conjunto con la alúmina forma el esqueleto de estas formulaciones una vez sinterizadas. Los tamaños de partículas están comprendidos entre $1-60 \mu \mathrm{m}$.

\subsubsection{FORMULACIÓN Y PREPARACIÓN DE LAS PASTAS CERÁMICAS}

La formulación empleada en la pasta original consistió en suspender las partículas de las materias primas en agua hasta lograr una solución homogénea con la siguiente relación por- centual en peso: $50 \%$ de alúmina, $25 \%$ de arcilla y $25 \%$ de cuarzo. Uno de los factores importantes para controlar la porosidad y tamaño de poro de las membranas a obtener está en función directa con el tamaño de las partículas que forma la pasta cerámica. La molienda húmeda permite, una vez realizada la formulación y preparación de la pasta inicial, variar el tamaño de partículas en función del tiempo empleado.

La pasta original, designada M00, fue sometida a molienda en un molino planetario. Se trabajó manteniendo constante su velocidad de giro, 200 r.p.m. Los tiempos de moliendas seleccionados fueron de 40, 20, 10 y 1 hora, que originaron cuatro diferentes pastas denominadas como M01, M03, M06 y M09 respectivamente. La distribución de tamaños de partículas de la pasta original y de aquellas sometidas a molienda se determinaron por rayos $X$ en un equipo Sedigraph. Los resultados se encuentran representados en la Figura 1, siendo los diámetros medios de partículas de cada una de las pastas los siguientes: 52,32 $\mu \mathrm{m}$ (M00); 2,54 $\mu \mathrm{m}$ (M01); 5,14 $\mu \mathrm{m}$ (M03); 6,48 $\mu \mathrm{m}$ (M06) y 10,23 $\mu \mathrm{m}$ (M09).

Para obtener una buena dispersión, homogeneidad y plasticidad de la pasta, la suspensión fue ajustada con el agregado de defloculantes (sulfato de sodio y ácido tánico). El agregado de electrólitos es controlado mediante medidas de viscosidad de la barbotina, hasta formar una suspensión tixotrópica-pseuplástica. Los valores de viscosidad estuvieron comprendidos entre 500$9000 \mathrm{mPa} \cdot \mathrm{s}$, con densidades próximas a $1,3 \mathrm{~g} / \mathrm{cm}^{3}$, (5).

\subsubsection{FABRICACIÓN DE LAS MEMBRANAS}

\subsubsection{Moldeado}

Se prepararon membranas planas de sección circular diámetro $(2.5 \pm 0.1) \cdot 10-2 \mathrm{~m}$ y espesor $(0.25 \pm 0.01) \cdot 10-2, \mathrm{~m}$ por el método de colada. Para ello se vierte la barbotina con apropiadas propiedades reológicas en moldes de yeso, donde la doble acción de eliminación de agua y floculación hace que la pasta se endurezca. Seguidamente la pieza colada se contrae separándose de las paredes del molde, (6).

\subsubsection{Secado}

Las muestras fueron posteriormente sometidas a secado, regulando la velocidad de evaporación de agua, para controlar de una forma secuencial la eliminación de agua libre contenida en los poros existentes entre las partículas y en la superficie de la muestra (temperatura ambiente hasta $110^{\circ} \mathrm{C}$ ).

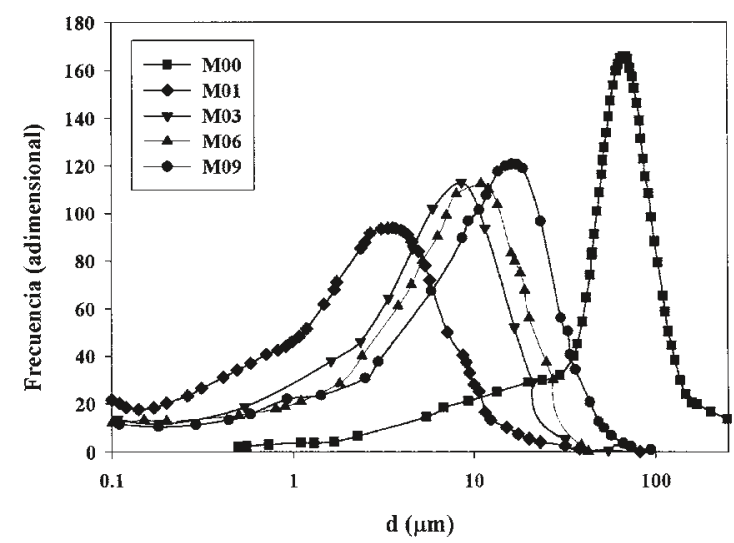

Fig. 1. Distribución de tamaños de partículas para las pastas: M00, M01, M03, M06, y M09. 


\subsubsection{Sinterizado}

Un tratamiento programado de temperatura permite controlar la evolución textural así como también la resistencia mecánica de las muestras sometidas al sinterizado. De los distintos componentes de nuestras pastas, el cuarzo con su transición $\alpha$ a $\beta$, es el que presenta la alteración polimórfica más importante, a $573^{\circ} \mathrm{C}$. En esta transición, se produce un $2 \%$ de expansión o contracción cúbica. El proceso de sinterizado se realizó en tres rampas de temperaturas. La primera desde $110^{\circ} \mathrm{C}$ hasta $600^{\circ} \mathrm{C}$ con una velocidad de calentamiento suave durante la cual se produce la eliminación del agua adsorbida y la transformación del cuarzo, una segunda etapa con una velocidad más pronunciada hasta alcanzar la temperatura de sinterizado, para finalmente mantener esta temperatura constante durante dos horas. Las temperaturas de sinterizado final de las pastas fueron 1200, $1300 \mathrm{y}$ $1400^{\circ} \mathrm{C}$

De esta forma, con los cuatro tipos de pastas empleados correspondientes a las cuatro distribuciones de partículas mencionados anteriormente y las tres temperaturas de sinterizado, obtendremos 12 membranas cuya nomenclatura se recoge en la tabla I.

\subsection{Métodos de caracterización}

Con el fin de determinar la estructura porosa de estas membranas, se han utilizado dos métodos de caracterización: Porosimetría de mercurio y la porometría de desplazamiento de gas-líquido. La diferencia, y por tanto la complementariedad de estos dos métodos, se basa en el tipo de poros que es capaz de detectar cada una de estas técnicas. La porosimetría de $\mathrm{Hg}$ nos proporciona información sobre la distribución total de poros dentro de la membrana y de su porosidad. Cuando hablamos de distribución total, nos referimos tanto de los poros activos (poros abiertos al flujo, es decir que permiten que un fluido atraviese la membrana cerámica), como de los poros no activos (huecos que contribuyen a la porosidad de la membrana, pero acaban en una barrera, de forma que no permiten el paso de un fluido). Por el contrario, la porometría de gas-líquido, proporciona la distribución de poros activos. Factor muy importante en los materiales porosos destinados a procesos de separación $(7,8)$.

\subsubsection{POROSIMETRÍA DE MERCURIO}

Se ha utilizado un porosímetro de mercurio Autopore III 9410 de Micromeritics ${ }^{\circledR}$. Este equipo permite el análisis de la distribución de tamaños de poro así como su contribución a la porosidad total, para poros comprendidos en el rango de 0.003 a $360 \mu \mathrm{m}$, lo cual corresponde a presiones aplicadas de hasta 60,000 psia $\left(4,14 \cdot 10^{6} \mathrm{~Pa}\right)$. El rango de trabajo elegido es analizado de forma automática por el equipo una vez alcanzadas las condiciones estáticas para cada presión o tamaño de poro equivalente. Es imprescindible un conocimiento lo más exacto posible del ángulo de contacto mercurio-material de membrana. Este ángulo podría ser medido directamente sin embargo, para ello sería preciso disponer de láminas totalmente planas y no porosas, hechas del mismo material que el que forma la membrana. Como eso es imposible en la mayoría de los casos, lo que se hace es utilizar un valor estándar que se ha mostrado válido para una gran cantidad de membranas de análogas características; en este caso se ha utilizado un valor de $130^{\circ}$.

\subsubsection{POROMETRÍA DE AIRE-LÍQUIDO}

Las determinaciones de porosimetría de aire-líquido se realizaron en un equipo Coulter $^{\circledR}$ Porometer II, fabricado por Coulter Electronics Ltd. Este aparato usa la técnica de desplazamiento de líquidos. Para ello la muestra se empapa completamente en un líquido (Coulter ${ }^{\circledR}$ Porofil) de baja tensión superficial $\left(\gamma=16 \cdot 10^{-3} \mathrm{~N} / \mathrm{m}\right.$ ), baja presión de vapor (3 mm Hg a 298 K) y baja reactividad. Se asume que el líquido rellena la totalidad de los poros presentes en la membrana, dado que tiene un ángulo de contacto prácticamente nulo con la casi totalidad de materiales usualmente encontrados en tecnología de membranas. A la muestra húmeda se le aplican entonces presiones crecientes a través de una fuente de aire limpio y seco, a $313 \mathrm{~K}$. Así al aumentar la presión del aire se alcanza un punto en el que dicha presión es suficiente para sobrepasar la tensión superficial del líquido en el interior de los poros más grandes, de forma que el líquido es expulsado. Si continuamos incrementando la presión, se irán vaciando poros de tamaños cada vez más pequeños a través de los cuales comenzará a fluir aire, de forma que un análisis del flujo de aire en función de la presión nos permita detectar la presencia de poros comprendidos entre 0,05 y $300 \mu \mathrm{m}$.

\section{RESULTADOS}

\subsection{Porosimetría de Mercurio}

A la vista de los resultados obtenidos por porosimetría de $\mathrm{Hg}$, lo primero que se observa es que existen dos poblaciones de poros, una con valores entre 10 y $0.2 \mu \mathrm{m}$ aproximadamente y otra por debajo de $0.1 \mu \mathrm{m}$. Es decir, la distribución de poros totales es bimodal, aunque desde el punto de vista de la microfiltración los poros por debajo de $0,1 \mu \mathrm{m}$ son de poca importancia para nuestro estudio.

Si analizamos el efecto de la distribución de las partículas empleadas en la preparación de la pasta, se observa que para las tres temperaturas de sinterizado, el máximo de la distribución se desplaza a tamaños mayores de la misma forma que lo hace la distribución del tamaño de partículas. Como un ejemplo, en la Figura 2, se muestran las distribuciones obtenidas para la temperatura de $1200{ }^{\circ} \mathrm{C}$. La distribución de poros por debajo de $0.1 \mu \mathrm{m}$ es similar para todas las pastas.

Cuando las membranas formadas con la misma pasta origi-

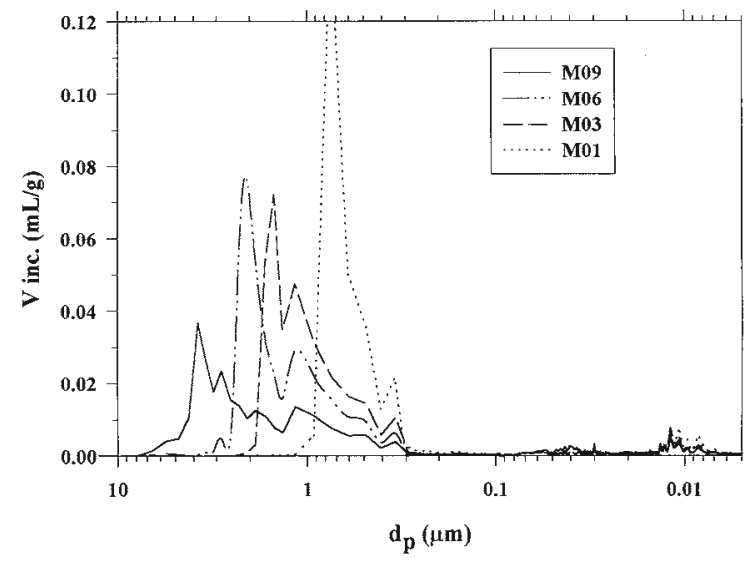

Fig. 2. Distribución de tamaños de poro obtenidas por porosimetría de $\mathrm{Hg}$ para cuatro membranas fabricadas con las pastas M01, M03, M06 y M09 y temperatura de sinterizado de $1200{ }^{\circ} \mathrm{C}$. 
nal se someten a distintas temperaturas de sinterizado, la tendencia observada en los porogramas de penetración de $\mathrm{Hg}$ al aumentar la temperatura, es un desplazamiento a tamaños mayores de poro, por aparición de poros de mayor tamaño. Además, es de resaltar que superpuesto a este ensanchamiento de la distribución (el extremo de poros menores no se desplaza de $0.2 \mu \mathrm{m}$ ), el cambio va acompañado de una disminución de la altura del pico por el efecto de la temperatura. Esto se puede observar en la Figura 3 para las membranas fabricadas con menores tamaños de partícula.

Respecto a los resultados de porosidad de las muestras, los valores en todos los casos son próximos al 50\%, aunque se observa una tendencia a disminuir con el aumento de la temperatura de sinterizado.

\subsection{Porometría de aire-líquido}

Los resultados de poros activos por porometría de airelíquido, muestran un comportamiento similar a la porosimetría de $\mathrm{Hg}$. Respecto al efecto de la distribución de partículas de la pasta original, se observa que las distribuciones con tamaño menores proporcionan membranas con poros activos menores y distribuciones de estos más estrechas. Esto se puede observar en la Figura 4, para la temperatura de 1200 ${ }^{\circ} \mathrm{C}$.

La influencia de la temperatura produce un aumento significativo del tamaño de poro, pero más por ensanchamiento de la distribución a valores de poros grandes que por corrimiento de éstas. En la Figura 5 se puede observar este efecto para las mismas membranas que en la Figura 3.

\section{DISCUSIÓN}

Los dos métodos de caracterización empleados presentan las mismas tendencias en las distribuciones de tamaños de poro: Aumento del tamaño de poro con el tamaño de partícula de la pasta empleada, y para un mismo tamaño de partícula, incremento del tamaño de poro con la temperatura. Los resultados del tamaño medio obtenidos por las dos técnicas se encuentran recogidos en la Tabla I. El efecto de la temperatura, produce un ensanchamiento de la distribución hacia poros de mayor tamaño. Esto se puede observar en la Figura 6 en los resultados de porometría aire-líquido, donde se presentan los valores medios de estas distribuciones así como su anchura representada por la desviación estándar. Es evidente que estos efectos de la temperatura deben estar relacionados con el crecimiento, durante el proceso de sinterizado, del sistema de partículas que forman la pasta; y que debe estar vinculado al tamaño y la composición química de éstas.

Las teorías concernientes al sinterizado, versan sobre el transporte de masa hacia la posición de contacto entre dos partículas y la cinética asociada con diferentes fenómenos de transporte. Aunque estas teorías están limitadas a la primera etapa de crecimiento de un área de contacto, parecen ser consistentes con observaciones experimentales concernientes a ordenamientos lineales de partículas esféricas idénticas. Por ejemplo, las teorías sugieren que la cinética de densificación estaría en relación inversa al tamaño de partícula (9). Sin embargo, partículas de tamaño extrafino pueden ser más difíciles de sinterizar que aquellos polvos más gruesos con químicas similares. La aplicación de estas teorías a un sistema como el nuestro, donde el tamaño, forma y composición química son diferentes, no sería apropiada para lograr explicar el fenómeno de sinterizado y su influencia en el tamaño de poro de las membranas cerámi-

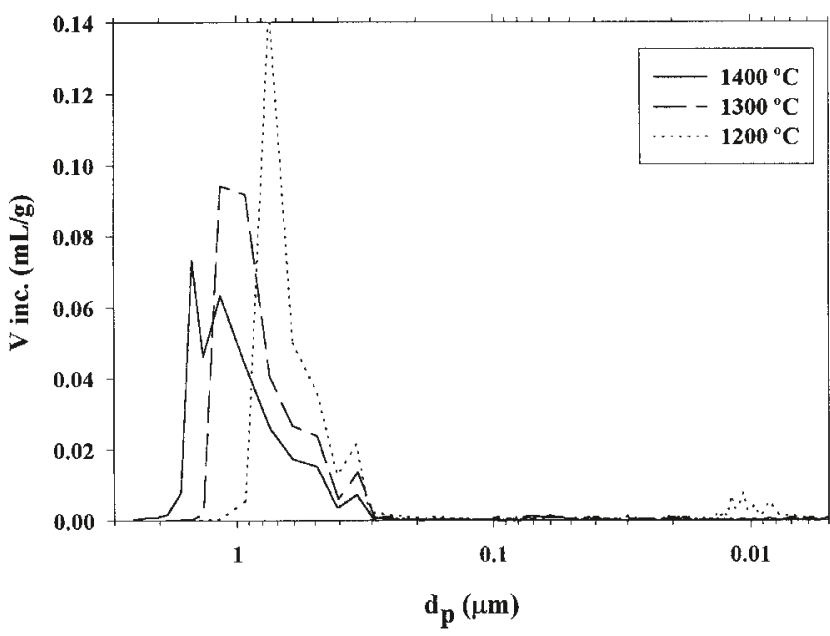

Fig. 3. Distribución de tamaños de poro obtenidas por porosimetría de $\mathrm{Hg}$ para membranas fabricadas con la pasta M01 y diversas temperaturas de sinterizado.

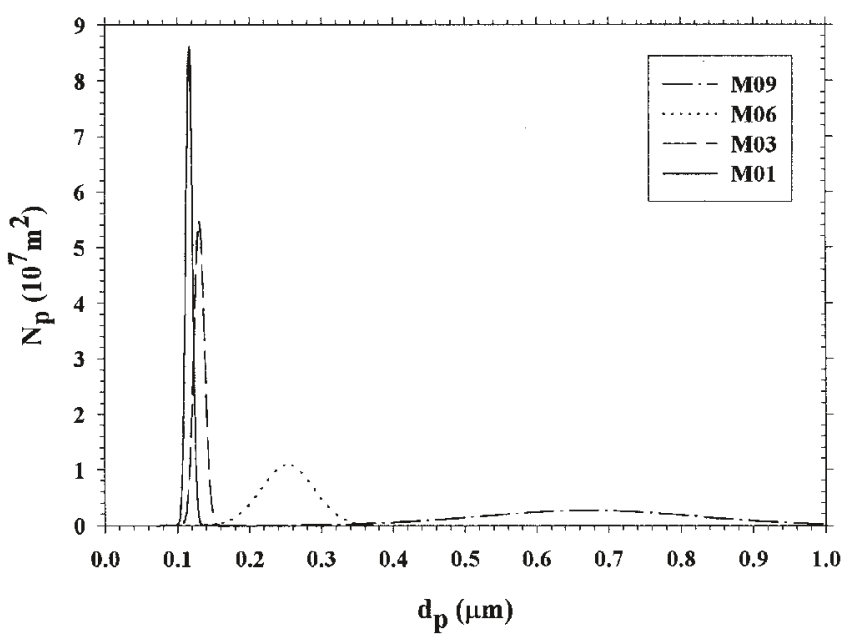

Fig. 4. Distribución de tamaños de poro obtenidas por porometría aire-líquido para cuatro membranas fabricadas con las pastas M01, M03, M06 y M09 y temperatura de sinterizado de $1200^{\circ} \mathrm{C}$.

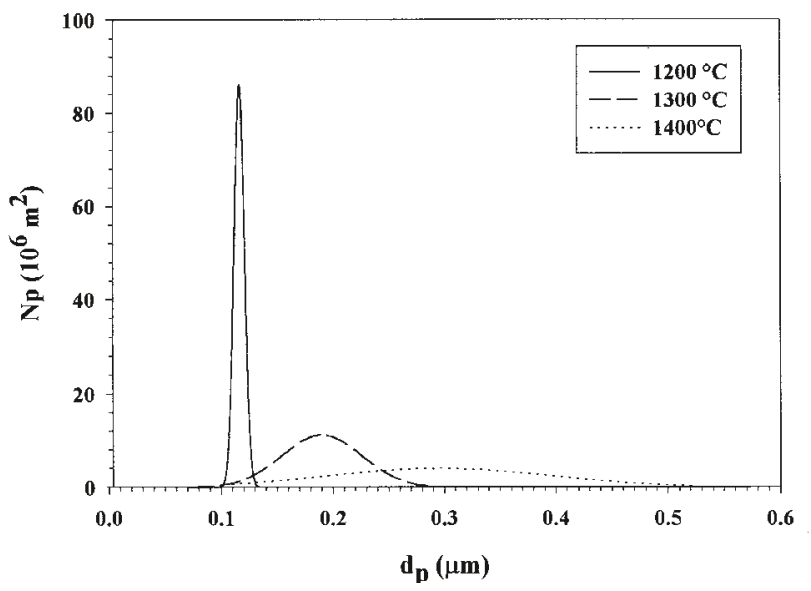

Fig. 5. Distribución de tamaños de poro obtenidas por porosimetría de $\mathrm{Hg}$ para membranas fabricadas con la pasta M01 y diversas temperaturas de sinterizado. 


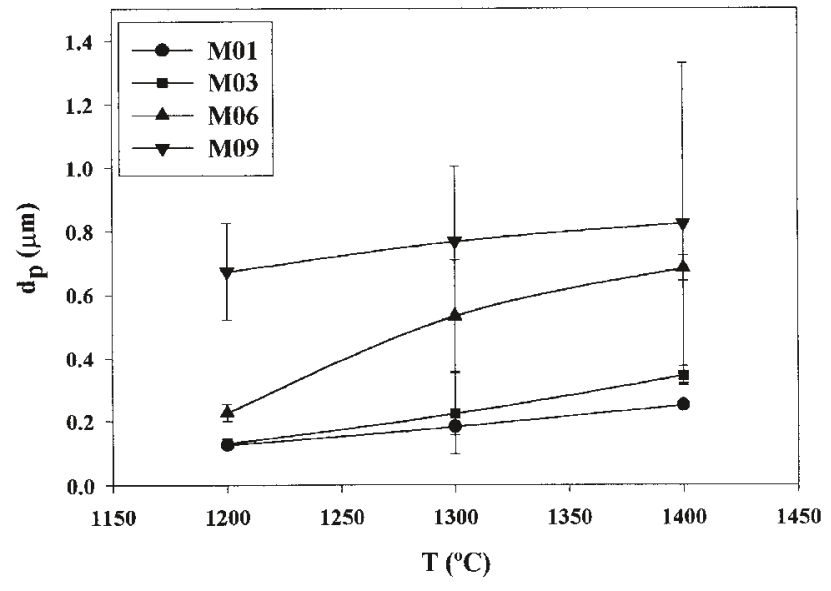

Fig. 6. Valores medios de la distribución de tamaños de poro, en función de la temperatura para las membranas estudiadas. El ancho de la distribución está representado con líneas verticales utilizando la desviación estándar de la distribución.

cas obtenidas. A fin de interpretar el comportamiento observado en nuestras membranas, se ha propuesto explicar de forma cualitativa los cambios secuenciales que podrían sufrir con la temperatura el conjunto de partículas constituyentes de la pasta, desde su estructura cruda hasta el sinterizado final.

Para simplicidad del tratamiento, consideremos que el sistema está compuesto de partículas esféricas con dos tamaños diferentes. Las etapas secuenciales de los efectos de quemado sobre la estructura del material cerámico se encuentran esquematizadas en la Figura 7.

$\mathrm{Al}$ inicio del sinterizado $\left(1000-1100^{\circ} \mathrm{C}\right)$, el transporte de masa es impulsado por el decrecimiento en la energía libre de Gibbs asociada con la disminución del área interfacial externa (vaporsólido) de las partículas, que son sometidas al crecimiento del área de contacto (cuello). Esta etapa es conocida como la formación y crecimiento de límites granulares, en donde las partículas se ordenan por un arreglo de las esferas en contacto, formando anillos o poliedros. Estas formaciones cerradas o "clusters" contienen un poro simple, que con un incremento de la temperatura $\left(1200-1400{ }^{\circ} \mathrm{C}\right)$, producen una transferencia de masa hacia una configuración más estable, con un aumento en la resistencia mecánica (configuración monolítica). En esta etapa, algunos de los microporos formados por los "clusters" de partículas pequeñas pueden disminuir su tamaño así como desaparecer, o simplemente contraerse hasta un tamaño de equilibrio. Esto conlleva un aumento gradual de los macroporos conformados por el aglomerado de partículas más grande, sin una variación apreciable en el volumen de poros. Este com-
TABLA I. DESCRIPCIÓN DE LAS MEMBRANAS UTILIZADAS, EN FUNCIÓN DE LAS CARACTERÍSTICAS DE FABRICACIÓN Y DIÁMETRO MEDIO DE PORO OBTENIDO MEDIANTE POROSIMETRÍA DE HG Y POROMETRÍA AIRE-LÍQUIDO. LA ÚLTIMA LETRA EN LA CLAVE DE CADA MEMBRANA REPRESENTA LA TEMPERATURA DE SINTERIZADO. $\mathrm{A}=1200{ }^{\circ} \mathrm{C}, \mathrm{B}=1300{ }^{\circ} \mathrm{C}$ Y $\mathrm{C}=1400{ }^{\circ} \mathrm{C}$.

\begin{tabular}{|c|c|c|c|}
\hline MEMBRANA & PASTA & $\begin{array}{c}\mathbf{d}_{\mathbf{p}}(\mu \mathrm{m}) \\
\text { Mercurio }\end{array}$ & $\begin{array}{c}\mathbf{d}_{\mathbf{p}}(\mu \mathbf{m}) \\
\text { Aire-líquido }\end{array}$ \\
\hline M01A & M01 & 0,66 & 0,12 \\
\hline M03A & M03 & 1,29 & 0,13 \\
\hline M06A & M06 & 1,85 & 0,23 \\
\hline M09A & M09 & 2,61 & 0,67 \\
\hline M01B & M01 & 0,69 & 0,18 \\
\hline M03B & M03 & 1,85 & 0,22 \\
\hline M06B & M06 & 3,15 & 0,53 \\
\hline M09B & M09 & 3,30 & 0,77 \\
\hline M01C & M01 & 1,21 & 0,25 \\
\hline M03C & M03 & 2,14 & 0,34 \\
\hline M06C & M06 & 3,60 & 0,68 \\
\hline M09C & M09 & 6,95 & 0,82 \\
\hline
\end{tabular}

portamiento se observa en las Figuras 3 y 5, en donde para una pasta con la misma distribución de partículas, el aumento de temperatura produce un incremento en el tamaño medio de poro.

Es de suponer que tratamientos térmicos superiores a los $1400{ }^{\circ} \mathrm{C}$ producirá una acentuada contracción de la masa cerámica, acompañada por una densificación y una disminución del tamaño de poro y porosidad.

\section{CONCLUSIONES}

Como consecuencia de este trabajo, se puede establecer una serie de conclusiones que se resumen en los siguientes enunciados:

1) Las membranas cerámicas de microfiltración, que han sido sintetizadas, poseen características de comportamiento similares a las membranas cerámicas comerciales.

2) La utilización de dos métodos complementarios posibilita la determinación de la estructura, tanto en poros totales como en aquellos abiertos al flujo.

3) El tamaño de poros activos, que son los que caracterizan principalmente las propiedades de separación de la membrana, viene determinado por la distribución del tamaño de partículas que forman la pasta.

Para una distribución de partículas determinada, las propiedades de la distribución de tamaños de poro de la membrana pueden ser modificada (tanto el valor medio de la distribución como la anchura de esta), en función de la temperatura de sinterizado.
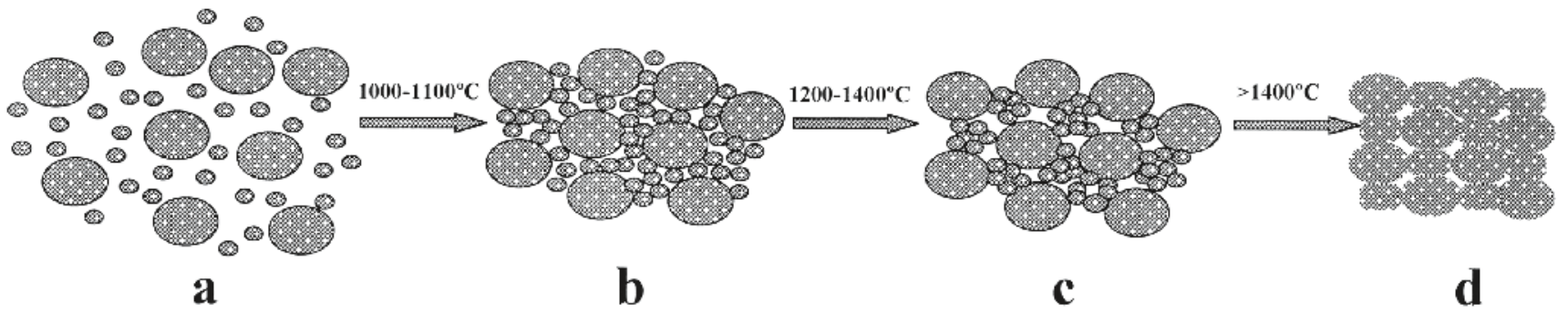

Fig. 7. Esquema de las variaciones de la estructura del material producidas por los efectos de quemado: a) Pasta cruda, b) Formación y crecimiento de límites granulares, c) Configuración monolítica y d) Densificación y disminución de la porosidad. 
Como tendencia general, en el entorno de $1100-1400{ }^{\circ} \mathrm{C}$, se puede afirmar que una mayor temperatura de sinterizado produce un desplazamiento de la distribución a tamaños de poro mayores y un ensanchamiento de ésta.

El efecto de aumento de tamaño de poro con la temperatura de sinterizado parece estar determinado por la coexistencia de un gran número de partículas pequeñas junto a las partículas grandes. El crecimiento de estas partículas de menor tamaño dentro de la estructura de las grandes, es el que provoca un aumento de la distribución de tamaños de poro.

\section{AGRADECIMIENTOS}

Los autores argentinos agradecen el apoyo de la Agencia Nacional de Promoción Científica y Técnica (ANPC y T-PICT97-N¹4-00013-00267) y Consejo Nacional de Investigaciones Científicas y Técnicas (CONICET-PIP No354/98). Los autores españoles agradecen la financiación de los siguientes proyectos de Investigación: QUI96-0767 del "Plan Nacional de Investigación y Desarrollo" (CICYT) y VA10496 de la Junta de Castilla y León.

\section{BIBLIOGRAFÍA}

1. R. Rautenbach y R. Albrecht, “Membrane Processes”, John Wiley and Sons, Nueva York, EE.UU., (1989).

2. R.R. Bhave, "Inorganic Membranes: Synthesis, Characteristics and Applications", Van Nostrand Reinhold, Nueva York, EE.UU., (1991).

3. K. Scott, "Handbook of Industrial Membranes", Elsevier, Oxford, Gran Bretaña (1995)

4. W.D. Kingery, H.K. Bowen y D.R. Uhlmann, "Introtuction to Ceramics", John Wiley and Sons, Nueva York, EE.UU., (1975).

5. C. Almandoz, "Preparación y Caracterización de Capas Activas de Aluminosilicatos para Microfiltración", Tesis para optar al Grado de Magister, Universidad de San Luis, San Luis, Argentina, (1998).

6. J. Marchese, "Membranas: Procesos con Membranas", Editorial Universitaria de San Luis, San Luis, Argentina, (1995).

7. A. Hernández, J.I. Calvo, P. Prádanos y L. Palacio, “A Multidisciplinary Approach Towards Pore Size Distributions of Microporous and Mesoporous Membranes", en Surface Chemistry and Electrochemistry of Membranes, Marcel Dekker inc., New York, (1999).

8. A. Hernández, P. Prádanos, J.I. Calvo, and L. Palacio, "Metal Oxide Membranes", en Oxide Surfaces, (Ed. J.W. Wingrave), Marcel Dekker, Nueva York, EE.UU., en Prensa.

9. F.F.Lange and B. Kellett, "Influence of Particle Arrangement on sintering", Science of Ceramic Chemical Processing, (Eds. L.L. Hench and D.R. Ulrich), John Wiley and Sons, Nueva York, EE.UU. (1986). 